The medicalization of life and society: origin, participants and consequences

\title{
La medicalización de la vida y la sociedad contemporánea: origen, participantes y consecuencias
}

\author{
Jesús Parra Sáez \\ Universidad de Murcia \\ jesus.parra@um.es
}

DOI: https://doi.org/10.15366/bp2019.22.011

Bajo Palabra. II Época. № 22. Pgs: 221-238

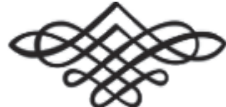


Recibido: 09/01/2017

Aprobado: 02/10/2019

\section{Resumen}

La amplia definición de salud establecida por la OMS en 1946 inició la extensión del área de actuación de la medicina hasta englobar los distintos ámbitos que constituyen la vida de las personas -economía, política, sociedad-, estableciendo la identificación entre los términos de «salud» $\mathrm{y}$ «bienestar». Como resultado, emergió un fenómeno denominado como «medicalización de la vida y la sociedad» en el que hay tres participantes fundamentales: los pacientes, los médicos y la industria farmacéutica. En la actualidad, el fenómeno de la medicalización ha acabado por desarrollar una concepción de la salud caracterizada como un próspero negocio internacional.

Palabras clave: Bienestar; OMS; Medicalización; Negocio; Crisis Sanitaria..

\section{Abstract}

The wide definition of health established by the WHO in 1946 started the extension of the action area of medicine to encompass the different areas that make up the lives of people-economy, politics, society- establishing the identification between the terms "health" and "welfare». As a result, a phenomenon called "medicalization of life and society" emerged in which there are three fundamental participants: patients, doctors, and the pharmaceutical industry. $\mathrm{Cu}$ rrently, the phenomenon of medicalization has finally developed a conception of health characterized as a prosperous international business.

Keywords: Health; Welfare; WHO; Medicalization; Business; Health Crisis. 


\section{Introducción}

La ORGANIZACIÓN MUNDIAL DE LA SALUD (OMS) nació el 22 de Julio de 1946 en el seno de las Naciones Unidas (ONU) como institución "independiente de las decisiones políticas y de otras agencias, que reforzara los lazos entre los descubrimientos médicos y las necesidades de salud e incluyese la mayor cantidad de países posible, independientemente de su orientación política" ${ }^{1}$, esto es, como una agencia de salud con carácter internacional ${ }^{2}$ anexada a aquélla que tenía un objetivo primordial: lograr que todos los pueblos y civilizaciones humanas alcanzaran un nivel de salud lo más elevadamente posible. Siguiendo este principio, la OMS proclamó su definición de salud" : "la salud es un estado de completo bienestar físico, mental y social, y no solamente la ausencia de afecciones o enfermedades" ${ }^{4}$, llevando a cabo una identificación entre los términos «salud» y «bienestar».

En este artículo se pretende mostrar cómo dicha identificación hace que el término «salud» tal y como lo conocemos transcienda no sólo el aspecto físico de las personas, sino también el aspecto psicológico hasta agrupar todos los ámbitos de la vida y de la sociedad en los que se ve envuelta cualquier persona (economía, cultura, política...). Esta extensión de la salud -y por lo tanto de aquella ciencia que se ocupa de ella: la medicina- a todos los ámbitos de la vida, permite a la medicina ampliar su área de actuación para intervenir en campos decisivos de las personas, teniendo como resultado un fenómeno generalmente denominado como «medicalización de la vida y de la sociedad».

Al tiempo, no sólo se da la medicalización de la vida (en el estricto sentido foucaultiano ${ }^{5}$ que tiene su inicio en el siglo XVIII), sino que se da una medicalización

${ }^{1}$ Cueto, $\mathrm{M}$ et al, "El proceso de creación de la Organización Mundial de la Salud y la Guerra Fría", en Apuntes. Revista de Ciencias Sociales, 38 (69), 2011, p. 133.

2 La inclusión de la definición de salud en la Constitución de la OMS implicó la aceptación tácita por parte de sus Estados miembros - caracterizados por su diversidad ideológica, cultural e idiosincrática- de un significado unitario de salud para todos.

3 La definición de salud fue promulgada por la OMS en la firma de su constitución el 22 de Julio de 1946 y entró en vigor el 7 de Abril de 1948. Hasta el día de hoy dicha definición sigue vigente.

4 "Constitución de la Organización Mundial de la Salud». Documentos básicos 45a Edición Suplemento, $2006,1$.

5 "La medicalización, es decir, el hecho de que la existencia, la conducta, el comportamiento, el cuerpo humano, se incorporaran a partir del siglo XVIII en una red de medicalización cada vez más densa y amplia, que cuanto más funciona menos se escapa a la medicina". Foucault, $M$, "Historia de la medicalización", en Educación médica y salud, 1, 1977, p. 4. 
ampliada que fomenta la aparición de un alto porcentaje de personas (especialmente en países desarrollados) que son subsidiarias de un tratamiento o de una medicación determinada en gran parte de sus vidas. Esto ocurre al identificar la enfermedad con toda una serie de situaciones sociales y personales (por ejemplo el malestar social de los desempleados o de los matrimonios fuertemente afectados por una crisis económica) que antes poco o nada tenían que ver con una patología que precisara de tratamiento médico. Esto promoverá con el paso de las décadas, generaciones de personas que no serán capaces de concebir la vida sin tomar un buen número de fármacos que finalmente constituirán -de hecho ya lo están haciendo- un negocio multimillonario en el que participan numerosos agentes entre los que destacan la industria farmacéutica y los propios profesionales de la medicina, especialmente los médicos, emergiendo un fuerte conflicto ético ${ }^{6}$. Finalmente, hay quien va más allá y manifiesta que la crisis actual del sistema sanitario es debida a la evidente medicalización de la vida y a la dimensión de injusticia ${ }^{7}$ que caracteriza a ésta.

\section{Origen de la medicalización contemporánea}

EL FENÓMENO QUE EN LA ACTUALIDAD conocemos como «medicalización de la vida y la sociedad" tiene su origen inmediato en la definición de salud establecida por la OMS en el año 1946, pero ¿cómo ha llegado la OMS hasta esa definición? El término salud no siempre ha englobado el aspecto social de las personas, sino que ese hecho se ha dado de forma relativamente reciente.

En la Antigua Grecia la noción de «salud» era identificada con la justicia, con la pureza y con la belleza, otorgándole el significado de un "estado deseable" para los hombres, y al mismo tiempo, el orden de la naturaleza humana. Posteriormente, en la época moderna -especialmente durante el siglo XVII- se pasa del hombre entendido como ser dentro de la naturaleza, a ser considerado como un ser que trasciende esa naturaleza. Se comienza a concebir al hombre como ser moral, como ser libre y como ser que tiene la tarea de dominar los fenómenos naturales. En esta época la salud se identifica con el derecho. Ahora bien, no se sustituye la justicia por el derecho, sino que el Estado debe distribuir, en la medida de lo posible, ese derecho que tienen los ciudadanos de acceso a la salud de una forma igualitaria (justicia distributiva). Entre el siglo XVIII y el XIX la

\footnotetext{
${ }^{6}$ Véase: Gómez Córdoba, A et al, "Dilemas éticos en las relaciones entre la industria farmacéutica y los profesionales de la salud”, en Persona y bioética, 11 (1), 2007, pp. 23-38.

7 Injusticia en tanto que deficiencia a la hora de distribuir los limitados servicios médicos.
} 
salud empieza a entenderse desde una tercera perspectiva: la social. Así, cuando se habla de la vida y también de salud, se hace en términos naturales, morales y sociales. Ya en el primer tercio del siglo XX y de forma restringida, algunos Estados -Reino Unido o Estados Unidos- comienzan a entender diversos fenómenos sociales como el alcoholismo, la delincuencia e incluso la indigencia, como patologías genético-hereditarias de las que la medicina habría de ocuparse y en virtud de las cuales un individuo sería considerado como un enfermo. Un buen ejemplo es el representado por el denominado Comité Wood (1924) en Reino Unido, el cual incluyó a numerosas personas con una situación económico-social precaria en su listado -el conocido como Informe Wood de 1929- de personas con deficiencias mentales ${ }^{8}$ sin que hubiera pruebas de que padecieran tales deficiencias.

La crudeza de la II Guerra Mundial supuso un punto de inflexión en la concepción de la salud, tanto por parte de las instituciones médicas como por parte de la sociedad en general. A partir de dicho momento tuvo lugar lo que el historiador Eric Hobsbawm denomina "revolución social", en la que la salud adquirió una importancia vital, más si cabe teniendo en cuenta las consecuencias de la guerra para la vida de las personas, especialmente las que estuvieron inmersas en la batalla directa (soldados) o indirectamente (familiares). De este modo, en la segunda mitad del siglo XX la preocupación general por la salud de las personas se maximizó -como contraposición al escaso valor que tiene una vida en un conflicto armado- y en el seno de los países más desarrollados emergió el denominado "Estado de Bienestar", en el que se busca el bienestar pleno de todos los ciudadanos de una sociedad. Fue aquí precisamente donde tuvo lugar el último cambio de significado en la concepción de salud, al establecerse una identificación salud-bienestar dando como resultado la definición manifestada por la «Organización Mundial de la Salud» en 1946. Tal identificación da pie a que la medicina abarque un gran número de ámbitos y que lo haga con un poder enorme:

La medicina se convierte en un nuevo poder, capaz de dictaminar lo normal, lo bueno y lo justo en diversos ámbitos, pues casi todos ellos, en una u otra perspectiva, pueden considerarse relacionados con la salud, cuando ésta es concebida de un modo tan amplio. Y, puesto que la enfermedad es universal, este poder se cree también con alcance para toda la humanidad ${ }^{10}$.

\footnotetext{
${ }^{8}$ Véase: Porter, D, "Eugenics and the Sterilization Debate in Sweden and Britain before World War II", en Scandinavian Journal of History, 24, 1999, pp. 145-162; y Blacker, C, "Voluntary Sterilization: The Last Sixty Years", en The Eugenics Review, 54 (1), 1962, pp. 9-23.

9 Véase: Hobsbawm, E, Historia del siglo XX, Editorial Crítica, Barcelona, 2011, Cap. X.

${ }^{10}$ Feito, L, "La definición de salud", en Diálogo filosófico 34, 1996, p. 74.
} 
La consecuencia directa de esta situación es la aparición de una realidad clara, una medicalización de la vida y la sociedad en la que "la sanidad crece de manera progresiva e indefinidamente, de manera que se superpone con el ámbito de la vida humana entera" ${ }^{11}$. A su vez, esta medicalización "crea" una serie de enfermedades o patologías que no lo son como tal, y para las cuales se desarrolla un tratamiento determinado que finalmente es adoptado de forma sistemática por los presuntos enfermos:

La definición sobre salud de la OMS de 1946, que considera a ésta de forma utópica y subjetiva como algo más que la ausencia de enfermedad y la eleva al estado de bienestar absoluto, contribuye a la incorporación al ámbito médico de nuevas situaciones y problemas. En la actualidad, situaciones de la vida como el cansancio o la frustración y variantes de la normalidad como la calvicie o la fealdad están siendo consideradas como enfermedades y, en muchos casos, sometidas a tratamiento farmacológico o incluso quirúrgico ${ }^{12}$.

Este tema es ampliamente tratado, y no son pocos los pensadores que se han hecho eco de él. Buenos ejemplos son los constituidos por Susana Rodríguez, y los doctores Morell Sixto, Martínez González y Quintana Gómez, quienes señalan a la creación de enfermedades enfocadas al crecimiento del consumo de fármacos como una de las primeras consecuencias del proceso de medicalización:

Sobre este tejido social, nace el concepto disease mongering (DM), término anglosajón difícil de traducir, que engloba aspectos como venta, invención y fabricación de enfermedades. La traducción que más se ajusta podría ser la de "mercantilización de las enfermedades", refiriéndose a la idea de obtener beneficios económicos fomentando la conciencia de enfermedad y la necesidad de medicinas para curarse. ${ }^{13}$

No sólo se trata de crear nuevas enfermedades que han de ser tratadas, sino que patologías consideradas leves hasta ahora pasan a ser consideradas como graves y de necesario tratamiento médico, incrementando al tiempo otro factor propio a la medicalización y que veremos más tarde, el económico: "hacer creer a la gente que está enferma puede ser una sustanciosa fuente de dinero. Una de las maneras de conseguir esto es ampliar los límites de las enfermedades tratables (...) por ejemplo, dolencias ordinarias en problemas médicos, leves síntomas en graves, problemas personales en problemas médicos, o convirtiendo riesgos en enfermedades". ${ }^{14}$

${ }_{11}$ García, D, El nacimiento de la bioética, Biblioteca Nueva, Madrid, 2007, p. 107.

12 Orueta, R, et al, "Medicalización de la vida (I)", en Revista Clínica de Medicina Familiar, 4, 2011, p. 151.

${ }_{13}$ Morell Sixto, M et al, "Disease mongering, el lucrativo negocio de la promoción de enfermedades", en Revista Pediatría de Atención Primaria, 11 (43), 2009, p. 493.

${ }^{14}$ Rodríguez, S, "El proceso de medicalización y sus consecuencias. Entre la moral, el poder y el negocio", en Intersticios, 2, 2008, p. 76. 
Como puede observarse, ni si quiera es necesario considerar enfermedades leves como graves, sino hacer descender los límites recomendados de indicadores como ha ocurrido con el colesterol, cuyos valores recomendados abarcan a un conjunto de la población escaso por lo que el resto de individuos son susceptibles de medicación enfocada al descenso de dichos indicadores.

\section{2. ¿Por qué dejar que la medicina se extienda a todos los ámbitos de la vida?}

Si ReAlmente Se tiene tan Claro que la medicina se ha extendido, y que aún lo sigue haciendo hacia toda una serie de ámbitos clave de la vida de las personas, ¿por qué no establecer unos límites? ¿Cuál es el motivo de dejar que se extienda la medicina a todos los ámbitos de la vida de una persona?

Hay quien establece una identidad entre lo que consideran que son los factores causantes de la medicalización y los factores que la mantienen en funcionamiento. Tizón señala a los procesos de duelo como uno de estos factores causantes, pero ¿a qué se refiere? Tradicionalmente, los individuos estaban acostumbrados a pasar el período de duelo tras una pérdida importante, más bien a aliviar sus penas, a través de medios estrictamente naturales como el llanto o las confidencias entre amigos. No obstante, con el paso de los años se ha tratado de reducir este período de duelo de forma drástica a través de toda una serie de tratamientos "antidepresivos", eliminando los estadios que acaban en la aceptación de la pérdida. Sin embargo, la mayoría de trastornos psicológicos asociados a la pérdida son de reciente existencia y el uso de fármacos para su solución parece tener un entorno económico más que clínico. Denomina a este fenómeno como «sufrimiento cronificado»:

Una parte cada vez mayor de nuestros conciudadanos en proceso de duelo, afectados por la turbulencia afectiva de haber perdido algo querido u odiado —o querido y odiado al tiempo-, va a ver cronificado su sufrimiento durante ańos. Y la forma más frecuente y extendida de cronificarlo es hoy el uso aventurero de todo tipo de psicofármacos, cuya utilidad no está probada en estas situaciones" ${ }^{15}$.

Por su parte, para Orueta y Cols la sociedad -la evolución del concepto de salud, una mayor valoración de la salud, la escasa educación sanitaria, o la disminución de los niveles de tolerancia-; los medios de comunicación -como fuente de información o por su poder mediático-; las instituciones políticas y sanitarias -al establecer servicios y organizaciones que se basan en demandas y no en necesidades, o la bu-

15 Tizón, J, “De los procesos de duelo a la medicalización de la vida”, en Jano, 1618, 2006, p. 59. 
rocratización sanitaria de la sociedad-; los profesionales - por convertir factores de riesgo en enfermedades o por su apoyo a la innovación terapéutica-; y la industria farmacéutica -al pretender expandirse en el mercado, al constituirse como una empresa, o por la promoción de enfermedades y los fármacos necesarios para combatirlas- son las verdaderas causas, tanto de la emergencia del fenómeno como de su mantenimiento en el futuro.

A mi parecer, el origen de la medicación tal y como la conocemos en la actualidad tuvo lugar en algunos fenómenos políticos, sociales y culturales -como la emancipación intelectual propia a la Ilustración, el desarrollo de la ciencia y la tecnología, o las consecuencias de dos guerras mundiales- que tuvieron como resultado la definición de salud establecida por la OMS, la cual al establecer la identidad salud-bienestar dio vía libre a que la medicina se extendiera al resto de ámbitos referentes al bienestar de las personas. Sin embargo, entiendo que los factores de los que se acaba de hablar aparecerían indudablemente como nexo de la definición de la OMS a la hora de promover y mantener la medicalización de la vida y de la sociedad. Susana Rodríguez comparte esta apreciación, y fecha en 1946 -fecha en que la OMS redefinió el término «salud»- el inicio del sistema de seguridad social actual en que la medicina se ocupa de satisfacer los estándares de bienestar de las personas:

La política de seguridad social está relacionada con el nuevo concepto de salud que propone la OMS en 1946 (...) A partir de este momento, las demandas e inversiones en salud se ven aceleradas ya que, de la curación y prevención, se pasa a la promoción de la salud. La medicina tiene que facilitar un bienestar para todos, lo que posibilita que entren en el mercado de la salud sin límites un ejército de expertos en cuerpos y almas que aplican diversas técnicas relacionadas con la autoestima y los modos de presentación. ${ }^{16}$

No obstante, entre los factores y/o participantes que mantienen la medicalización contemporánea hay tres especialmente notorios: los pacientes, la industria farmacéutica y los médicos. En primer lugar, no hay duda de que el ser humano es un ser de costumbres y no sólo eso, sino que es un ser al que le cuesta mucho desligarse de ellas. Este es el motivo principal por el que les es de gran dificultad a los individuos abandonar su "rutina médica", o quizás, su dependencia farmacológica. Desde que se da el fenómeno de la medicalización de la vida las personas están excesivamente medicadas, llegando al extremo de que prácticamente hay una píldora para cada molestia que se sufre o para elevar los niveles de cualquier vitamina, lo cual hace que las personas estén acostumbradas a una medicación diaria con

\footnotetext{
${ }^{16}$ Rodríguez, S, "El proceso de medicalización y sus consecuencias. Entre la moral, el poder y el negocio", op.cit., p. 75 .
} 
independencia de si están enfermas o no. Incluso se podría establecer una identidad entre persona y paciente, entonces ¿por qué no evitar esta excesiva medicación? ¿Por qué no evitar que las personas puedan identificarse con los pacientes? Porque las personas han vivido desde hace décadas de esta manera y no conciben una vida en la que por ejemplo, no puedan tomarse una aspirina para aliviar un dolor de cabe$\mathrm{za}^{17}$, o donde no haya un fármaco que combata la pérdida del cabello o los bajos niveles de hierro en la sangre:

Es llamativo observar cómo la dependencia del sistema sanitario y de los agentes de salud en general es cada vez mayor. Vivimos un cambio en la sociedad que ha modificado sus creencias y valores y en consecuencia también sus formas de considerar la enfermedad $\mathrm{y}$ de enfrentarse a los avatares normales de la vida. ${ }^{18}$

En segundo lugar, los mayores interesados en que se mantenga la medicalización de la sociedad son los laboratorios farmacéuticos que producen miles de millones de medicamentos y aparatos tecnológicos biomédicos en cadena para distribuir por todo el mundo, lo cual promueve la movilidad de una cantidad ingente de dinero que desemboca -en términos foucaultianos- en una verdadera «economía de la salud». Por ello la industria farmacéutica es la principal valedora de la "creación de enfermedades", esto es, establecer como patología a eliminar lo que antes sólo eran síntomas asociados a verdaderas patologías o simplemente eran deficiencias externas al ámbito de la salud. Además, se pretende que esto ocurra en ámbitos de carácter general donde pueda haber una demanda considerable del nuevo medicamento. La producción llega a extenderse casi a medicamento por síntoma y poco importa que sea o no una verdadera patología, o si los medicamentos de verdad benefician al individuo: "este camino conduce a una situación en que para cada nuevo diagnóstico o tratamiento se puede crear una enfermedad, con independencia del carácter de los beneficios que aquellos aporten" ${ }^{19}$. Para conseguir distribuir los fármacos que producen, las empresas farmacéuticas realizan una fuerte campaña publicitaria en la que se fomenta entre la población el miedo a patologías que, en la mayoría de las ocasiones, no son tal. Así, consiguen crear en las personas cierta necesidad de consumir sus medicamentos para evitar la patología en cuestión. Además de la

\footnotetext{
${ }_{17}$ El problema aparece principalmente en las personas que no quieren dejar de ser objeto de tratamiento y reclaman la producción de más y más medicamentos para patologías de escasa importancia o incluso para satisfacer una necesidad que nada tiene que ver con las enfermedades. Me atrevería a decir que, por lo general, los seres humanos - al menos los que vivimos en el Primer Mundo y gozamos de la posibilidad de adquirir este tipo de tratamientos médicos- somos unos "adictos" a los medicamentos.

${ }^{18}$ Morell Sixto, $\mathrm{M}$ et al, "Disease mongering, el lucrativo negocio de la promoción de enfermedades", op. cit., p. 492.

19 Márquez, S y Meneu, R, "La medicalización de la vida y sus protagonistas", en Eikasia. Revista de filosofía, 8, 2007, p. 75 .
} 
utilización de los medios de comunicación, en ciertas ocasiones se hacen tratos con médicos - de cabecera, psiquiatras- ${ }^{20}$ con el fin de que éstos consigan recetárselos a sus pacientes:

La independencia de los médicos se ha visto enormemente afectada. Por ejemplo, se ha comprobado que muchos de los autores que escriben de forma positiva sobre un medicamento reciben dinero de la industria (...) Algunos estudios de medicamentos no se llevan a cabo para aclarar cuestiones científicas, sino para colocar un medicamento en el mercado. El médico recluta entre su clientela objetos de ensayo para el estudio y les receta el medicamento en cuestión, por lo cual recibe una suma de la empresa ${ }^{21}$.

En tercer lugar los médicos, o lo que es lo mismo, el intermediario entre el paciente y la industria farmacéutica, se encuentran en una situación comprometida al debatirse entre guiar su actuación exclusivamente con la motivación de curar, y hacerlo con la motivación añadida de obtener algún beneficio externo. Por tanto, en ellos se da la unión del deseo de hacer el bien al paciente y de que se extienda el consumo de nuevas tecnologías biomédicas o nuevos derivados químico-farmacológicos: "las empresas farmacéuticas han realizado prácticas con la finalidad de conseguir mayor acercamiento y mejores ventas de sus productos en los pacientes (...) dejando al médico en un conflicto de intereses, que en muchas ocasiones ponen a prueba su ética. ${ }^{22}$ De este modo, hay ciertos casos en que los médicos se "alían" con las empresas farmacéuticas con las que se comprometen a distribuir los medicamentos que éstas producen a cambio de un incentivo social (mayor publicidad para el médico) o económico (obtención de dinero o de aparatos tecnológicos biomédicos) ${ }^{23}$. Aunque hay fuertes excepciones como la de Estados Unidos ${ }^{24}$

${ }^{20}$ Véase: Grande, D, "Limiting the Influence of Pharmaceutical Industry Gifts on Physicians: Self-Regulation or Government Intervention?”, en Journal of General Internal Medicine, 25 (1), 2010, pp. 79-83.

${ }^{21}$ Rodríguez, S, "El proceso de medicalización y sus consecuencias. Entre la moral, el poder y el negocio", op.cit. p. 79.

22 Buffo Sequeira, I et al, "El médico y la relación con la industria farmacéutica", en Revista CONAMED, 17 (4), 2012 , p. 183.

${ }^{23}$ En el film Efectos secundarios/Side effects dirigido por Steven Soderbergh (2013) se muestra esta situación. Se vislumbra cómo un psiquiatra distribuye un nuevo medicamento a sus pacientes a modo de estudio para una farmacéutica, obteniendo aquél cierta compensación económica y ofreciendo el fármaco de forma gratuita a sus pacientes para que acepten tomarlo. Al tiempo el film se hace eco de la medicalización de la vida y de la sociedad, especialmente en cuanto a la sociedad norteamericana se refiere.

${ }^{24}$ En Estados Unidos no hay una regulación que limite la publicidad - por parte de los colegiados- de unas marcas determinadas a la hora de recetar una medicación. En Espańa, por el contrario, y en virtud de la «ley 29/2006 de garantías y uso racional de los medicamentos y productos sanitarios», los médicos de la Seguridad Social únicamente pueden recetar el principio activo de un fármaco y no marcas concretas, lo cual limita el campo de acción de los visitadores médicos, esto es, personas que tratan de convencer al cuerpo médico para que recete unos fármacos en concreto. Además y en lo relativo al conflicto de intereses anteriormente mencionado, el artículo 3 recoge lo siguiente: "el ejercicio clínico de la medicina, de la odontología y de la veterinaria y otras profesiones sanitarias con facultad para prescribir o indicar la dispensación de los medicamentos será incompa- 
donde en el año 2003 y según IMS Health (organización estadounidense dedicada a publicar información relativa a al ámbito de la salud) la industria farmacéutica gastó 4.000 millones de dólares en material para promocionar sus medicamentos en las consultas médicas-, este hecho parece darse más comúnmente en aquellos lugares donde hay más desconocimiento sobre las diversas patologías y sus respectivos tratamientos, pues es mucho más sencillo para las farmacéuticas distribuir sus fármacos amparados por la opinión profesional de los médicos:

Las recomendaciones y consensos parten de las sociedades científicas y de los médicos líderes de opinión. Estas recomendaciones son especialmente relevantes en áreas con mayor incertidumbre, donde la guía de los expertos, los referentes profesionales, se configura como una de las fuentes primordiales de orientación ${ }^{25}$.

Al tiempo, la investigación biomédica está enfocada en la mayoría de los casos al cumplimiento de intereses de las empresas farmacéuticas, como muestra el hecho de que se investigue mucho más en tratamientos para frenar la calvicie o la disfunción eréctil que en tratamientos para luchar contra las denominadas "enfermedades raras", esto es, enfermedades que sólo afectan a un porcentaje mínimo de la población ${ }^{26}$ (menos de cinco por cada diez mil habitantes). Como afirman Stolkiner ${ }^{27}$ y Casas $^{28}$, quedan desamparados los individuos que padecen las «enfermedades huérfanas» $\mathrm{o}$ "enfermedades olvidadas», las cuales independientemente de su gravedad, no resultan rentables a las industrias y están fuera de la cobertura de los seguros, por lo que se acaba invirtiendo más tiempo y dinero en la búsqueda de un remedio para "falsas enfermedades" o para "enfermedades beneficiosas" - por el volumen de demandantes- que en investigación de este tipo de enfermedades:

Cuando la omisión de la industria farmacéutica (no prestar la atención necesaria a la investigación y desarrollo de nuevos productos efectivos para hacer frente a las EO) tiene como consecuencia la muerte de miles de personas cada año y la degradación de las condiciones de vida de muchas otras debido a la enfermedad, el debate sobre la ética de esta

tible con cualquier clase de intereses económicos directos derivados de la fabricación, elaboración, distribución y comercialización de los medicamentos y productos sanitarios”. Véase: Jefatura del Estado, «Ley 29/2006, de 26 de julio, de garantías y uso racional de los medicamentos y productos sanitarios», en: «BOE» núm. 178, de 27 de julio de 2006, disponible en: https://www.boe.es/buscar/pdf/2006/BOE-A-2006-13554-consolidado.pdf

25 Márquez y Meneu, "La medicalización de la vida y sus protagonistas”, op.cit. p. 74.

${ }^{26}$ La propia OMS ha afirmado que existen más de 7.000 enfermedades consideradas "raras" que afectan al 7\% de la población mundial, por lo que se están dejando millones de personas con patologías diagnosticadas sin tratar y sin interés por hacerlo debido al beneficio económico relativamente escaso que se obtendrá con ello.

27 Véase: Stolkiner, A, "Medicalización de la vida, sufrimiento subjetivo y prácticas en salud mental", en Los sufrimientos: 10 psicoanalistas 10 enfoques, Hugo Lerner (comp.), Psicolibro ediciones, Colección FUNDEP, 2013.

${ }^{28}$ Casas Roma, X, "Responsabilidad de la industria farmacéutica sobre la inversión en investigación de las enfermedades olvidadas. Propuesta de mejora”, en Daimon. Revista Internacional de Filosofía, 64, 2015, pp. 117-132. 
«omisión» debe ser, cuanto menos, planteado, discutido y argumentado de forma diligente por parte de los agentes implicados. ${ }^{29}$

Ahora bien, no se está señalando que los médicos no atiendan al bien de las personas, sino que dentro de ese afán por hacer el bien para el paciente se dan situaciones en las cuales algunos médicos no tienen claro si deben ceder o no ante las presiones de las farmacéuticas para distribuir nuevos fármacos o nuevas tecnologías biomédicas. En este sentido, Brennan y Cols en una comunicación de la «Asociación Médica Americana» (American Medical Association) advirtieron de la necesidad de establecer una regulación más estricta -en Estados Unidos $-{ }^{30}$ que "incluya la eliminación o modificación de prácticas comunes relacionadas con obsequios pequeños, muestras farmacéuticas, educación médica continua, fondos para viajes de médicos, oficinas de oradores, redacción fantasma y contratos de consultoría e investigación" 31 , esto es, el modus operandi de la industria farmacéutica para maximizar sus beneficios a través de los médicos, aprovechando su cercanía con los consumidores. En España, por suerte para nosotros y nuestros intereses exclusivamente médicos, la legislación vigente prohibe hacer publicidad o recetar marcas concretas de medicamentos: "deberán necesariamente presentarse y dispensarse bajo principio activo o, en su defecto, una denominación común o científica o la expresada en el formulario nacional y en ningún caso bajo marca comercial” ${ }^{32}$.

\section{3. ¿Es la crisis del sistema sanitario una consecuencia de la medicalización?}

Como se vio anteriormente, llegado el momento el concepto de salud comenzó a atender a la justicia en cuanto que distribución del derecho de salud. Sin embargo, la medicalización parece convertirse en una forma de injusticia, pues a mayor

\footnotetext{
29 Ibid., p. 121

30 Un buen número de instituciones médicas - por ejemplo la Asociación Médica Americana, el Colegio Americano de Médicos o el Consejo de Acreditación para la Educación Médica Continua- han analizado esta situación en territorio norteamericano. Sin embargo, las directrices o propuestas de actuación que han ofrecido para cambiar esta realidad no son lo suficientemente contundentes: "las pautas producidas por estos diversos grupos y organizaciones no son lo suficientemente estrictas y no mantienen adecuadamente un compromiso profesional con el bienestar del paciente y la integridad de la investigación. Ninguno de estos grupos establece mecanismos de monitoreo o identifica la responsabilidad del cumplimiento. La profesión misma debe ejercer un control mucho más estricto sobre las relaciones entre los fabricantes y los médicos". Brennan, T et al, "Health Industry Practices That Create Conflicts of Interest. A policy Proposal for Academic Medical Centers", en JAMA, 295 (4), 2006, p. 430.

31 Ibid. p. 429.

32 Jefatura del Estado, «Ley 29/2006, de 26 de julio, de garantías y uso racional de los medicamentos y productos sanitarios», en: «BOE» núm. 178, de 27 de julio de 2006, Cap. 4.
} 
número de personas sanas que precisan de medicación, menor será el volumen de medicación para los que de verdad la necesitan. Eso es lo que señala Pérez-Ciordia, la imposibilidad del sistema sanitario de satisfacer la demanda de los pacientes: "la amplia medicalización contribuye decisivamente a que los sistemas sanitarios ya no sean sostenibles" ${ }^{33}$. Precisamente lo que deriva de la medicalización contemporánea es el auge de la producción de fármacos, pues en la gran mayoría de los casos, lo que se demanda son medicamentos enfocados a paliar esas "enfermedades creadas" de las que ya se ha hablado. Por eso aboga por promover una verdadera concienciación de las personas acerca de lo que realmente constituye una patología o enfermedad, y lo que es una mera necesidad creada con el consumo como fin último. Esa concienciación es lo que él denomina "desmedicalización". Lo cierto es que en los últimos años -en España- el volumen de pacientes en los centros sanitarios ha crecido de forma diametralmente opuesta a la oferta médica. Los hospitales carecen cada vez más de personal y material sanitario, mientras los pasillos y salas de espera se abarrotan de enfermos. De este modo, la medicalización se erige como un factor -entre otros como los recortes en sanidad relativos a la crisis económica, el envejecimiento de la población, la privatización o el aumento del empobrecimiento de la población- ${ }^{34}$ que provoca la crisis actual del sistema sanitario.

\section{Conclusiones}

Tras el análisis del fenómeno de la medicalización de la vida y la sociedad contemporánea, sus causas y los agentes que la componen, se han alcanzado cuatro conclusiones principales:

1. El origen inmediato de la medicalización contemporánea es un conjunto de concepciones de "ser humano" cuya salud y bienestar transciende lo meramente físico-biológico y que se ven representadas por la definición de salud de la OMS de 1946 (Salud=Bienestar). Con el desarrollo del individuo y la sociedad, los diferentes ámbitos del ser humano -política, economía, sociedad, cultura- se han unido en torno a su salud convirtiéndose en condición de posibilidad para que ésta pueda darse. Más concretamente, la salud ha pasado de ser un concepto que alude al aspecto natural del hombre, a ser un concepto que engloba también al aspecto moral y social de éste. Este cambio

\footnotetext{
33 Pérez-Ciordia, I, “Las no enfermedades y la medicalización”, en Jano 1775, 2011, p. 76.

34 Véase: Martín Martín, J, “El sistema nacional de salud español ante la gran recesión”, en Cuadernos de Relaciones Laborales, 34 (2), 2016, pp. 315-334.
} 
tuvo lugar como consecuencia de una serie de fenómenos sociales como el movimiento ilustrado, el humanismo, y sobretodo todo lo que engloba a los dos grandes conflictos en que se ha visto envuelto el ser humano en el siglo $\mathrm{XX}$, las dos guerras mundiales. El poco valor que ha tenido la vida en general y la salud en particular en el desarrollo de la contienda -especialmente tras el descubrimiento del quehacer nazi durante el Holocausto-, es uno de los factores que hizo que de forma inmediata a su finalización las instituciones políticas y médicas internacionales dieran un nuevo sentido al término "salud», otorgándole una importancia especial y atendiendo a todo aquello que afecta al bienestar del individuo más allá del bienestar exclusivamente físico o fisiológico. Ese hecho queda reflejado en la definición de salud manifestada por la OMS en 1946 que establecía una identificación entre salud y bienestar, lo cual dio pie a una extensión de la medicina a todos los ámbitos de la vida y de la sociedad.

Así pues, el término salud ha sufrido una serie de transformaciones paralelas a las sufridas socialmente por el hombre. El desarrollo de un concepto de salud basado en el bienestar físico, o en su defecto, del bienestar físico-psicológico de las personas; hacia un concepto de salud que tiene el bienestar social como pilar fundamental junto al bienestar físico y mental; ha dado lugar a una definición de la salud que tiene como consecuencia directa la extensión de la medicina a los diferentes ámbitos de la vida. Al tiempo, la nueva concepción de salud se vería reforzada por toda una serie de factores como la fabricación en masa de fármacos por parte de la industria farmacéutica o la tendencia de los pacientes a medicarse en mayor medida de forma gradual, y en ocasiones, sin conocimiento de su médico de cabecera (auto-medicación).

2. El fenómeno de la medicalización se ha extendido en las sociedades desarrolladas hasta convertirse en un negocio que mueve miles de millones y que tiene como gran beneficiario a la industria farmacéutica. La identificación entre salud y bienestar, y el desarrollo posterior de la medicalización, han otorgado a la salud un cierto carácter comercial en la medida en que se ha convertido en un negocio millonario -especialmente para las grandes empresas farmacéuticas- que actúa siguiendo una política basada en la relación entre costes y beneficios, en la cual prevalece claramente lo económico respecto a lo sanitario. Fiel muestra de ello es que la investigación médica está enfocada a la creación de medicamentos de fácil distribución, esto es, de alta demanda; mientras que se abandona la investigación de enfermedades denominadas "raras" debido a su escasa demanda. 
Es innegable que la investigación médica está enfocada hacia el bien del paciente, pero de lo que no cabe duda es que dentro de ese bien al paciente, prevalece la investigación de la enfermedad del paciente que a más personas pueda llegar y que, en última instancia, más dinero pueda generar. Valga de ejemplo paradigmático una situación hipotética en la cual haya que investigar una enfermedad que no reviste gravedad, pero que afecta a un número considerable de personas; u otra enfermedad que sí es de gravedad, pero que afecta a un número mínimo de personas. En tal situación la investigación será enfocada hacia la producción de un medicamento que combata la enfermedad menos grave, pues a mayor demanda más producción de medicamentos y finalmente mayor beneficio para la empresa farmacéutica en cuestión.

3. Los pacientes son los primeros que se muestran reacios a acabar con el fenómeno de la medicalización. En las sociedades contemporáneas los pacientes, aun conociendo los problemas que puede conllevar auto-medicarse o incurrir en un exceso de medicación no conciben la vida sin ella. En las últimas décadas, en unos casos ha emergido y en otros ha sido creada en los pacientes -especialmente en lo de edad avanzada- la necesidad de ser subsidiarios de medicación aunque en muchas ocasiones no lo necesiten, o bien por no estar enfermo o porque su "dolencia" no consiste en una verdadera patología. La consecuencia directa de este fenómeno es que las personas se sienten progresivamente más dependientes de una medicación y, al mismo tiempo, se está fomentando - por parte de las farmacéuticas- que las personas no sepan realmente discernir entre lo que de verdad es un síntoma de una patología y lo que no: "estamos asistiendo a la generación de una dependencia de la medicina que incapacita a las personas para cuidarse a sí mismas y una disminución de la tolerancia al sufrimiento y al malestar" ${ }^{35}$. De esta forma las personas, o si se prefiere, los pacientes (prácticamente todos somos pacientes, pues la gran mayoría de las personas somos subsidiarias de alguna medicación), son los primeros en mostrarse reacios a poner límite al exceso de medicalización.

4. La medicalización no constituye (por sí sola) la causa directa de la crisis del sistema sanitario, aunque es uno de los factores que la refuerzan. Es cierto que un mayor volumen de demanda de medicamentos y de tratamientos puede colapsar el sistema sanitario en cuanto a la distribución del derecho a la salud, pero ¿qué ocurre con la falta de material sanitario?, ¿qué ocurre con

35 Orueta, R., et al, “Medicalización de la vida (I)”, op.cit. p. 151. 
la disminución de personal médico? El sistema sanitario se ve colapsado por diversos factores sociales y políticos, por ejemplo cuando se aplican una serie de recortes al ámbito de la sanidad que tienen como consecuencia una disminución considerable del material sanitario, o cuando se da un incremento de la esperanza de vida que da lugar a una sociedad donde residen un gran número de personas de elevada edad que estarán cada vez más necesitadas de una medicación. De este modo, la medicalización por sí sola no puede constituir la crisis del sistema sanitario, sino que son todo un conjunto de factores -entre los que se encuentra la medicalización- los que la conforman. 


\section{Referencias Bibliográficas}

Blacker, C, "Voluntary Sterilization: The Last Sixty Years", en The Eugenics Review, 54 (1), 1962, pp. 9-23.

Brennan, $\mathrm{T}$ et al, "Health Industry Practices That Create Conflicts of Interest. A policy Proposal for Academic Medical Centers", en JAMA, 295 (4), 2006, pp. 429-433. doi:10.1001/jama.295.4.429

Buffo Sequeira, I et al, "El médico y la relación con la industria farmacéutica", en Revista CONAMED, 17 (4), 2012, pp. 182-186.

Casas Roma, X, "Responsabilidad de la industria farmacéutica sobre la inversión en investigación de las enfermedades olvidadas. Propuesta de mejora”, en Daimon. Revista Internacional de Filosofía, 64, 2015, pp. 117-132. doi:10.6018/ daimon/173401

"Constitución de la Organización Mundial de la Salud». En: Documentos básicos 45ª Edición Suplemento, 2006.

Cueto, $\mathrm{M}$ et al, "El proceso de creación de la Organización Mundial de la Salud y la Guerra Fría", en Apuntes. Revista de Ciencias Sociales, 38 (69), 2011, pp. 129 156. doi:10.21678/apuntes.69.634

Feito, L, "La definición de salud", en Diálogo filosófico, 34, 1996, pp. 61-84.

Foucault, M, "Historia de la medicalización", en Educación médica y salud, 1, 1977, pp. 3-25.

García, D, El nacimiento de la bioética, Biblioteca Nueva, Madrid, 2007.

Gómez Córdoba, A et al, "Dilemas éticos en las relaciones entre la industria farmacéutica y los profesionales de la salud”, en Persona y bioética, 11 (1), 2007, pp. 23-38.

Grande, D, "Limiting the Influence of Pharmaceutical Industry Gifts on Physicians: Self-Regulation or Government Intervention?", en Journal of General Internal Medicine, 25 (1), 2010, pp. 79-83. doi:10.1007/s11606-009-1016-7

Hobsbawm, E, Historia del siglo XX, Editorial Crítica, Barcelona, 2011.

Jefatura del Estado, "Ley 29/2006, de 26 de julio, de garantías y uso racional de los medicamentos y productos sanitarios», en: «BOE» núm. 178, de 27 de julio de 2006. Disponible en: https://www.boe.es/buscar/pdf/2006/ BOE-A-2006-13554-consolidado.pdf 
Márquez, S y Meneu, R, "La medicalización de la vida y sus protagonistas", en Eikasia. Revista de filosofia, 8, 2007, pp. 65-86.

Martín Martín, J, "El sistema nacional de salud español ante la gran recesión”, en Cuadernos de Relaciones Laborales, 34 (2), 2016, pp. 315-334. doi: 10.5209/ CRLA. 53459

Morell Sixto, $\mathrm{M}$ et al, "Disease mongering, el lucrativo negocio de la promoción de enfermedades", en Revista Pediatría de Atención Primaria, 11 (43), 2009, pp. 491-512. doi: 10.4321/S1139-76322009000400011

Orueta, R., et al. "Medicalización de la vida (I)", en Revista Clínica de Medicina Familiar, 4, 2011, pp.150-161.

Pérez-Ciordia, I, "Las no enfermedades y la medicalización”, en Jano 1775, 2011, pp. 73-76.

Porter, D, "Eugenics and the Sterilization Debate in Sweden and Britain before World War II", en Scandinavian Journal of History, 24, 1999, pp. 145-162.

Rodríguez, $S$, "El proceso de medicalización y sus consecuencias. Entre la moral, el poder y el negocio", en Intersticios, 2, 2008, pp. 71-85.

Stolkiner, A, "Medicalización de la vida, sufrimiento subjetivo y prácticas en salud mental", en Los sufrimientos: 10 psicoanalistas 10 enfoques, Hugo Lerner (comp.), Psicolibro ediciones, Colección FUNDEP, 2013.

Tizón, J, "De los procesos de duelo a la medicalización de la vida", en Jano, 1618, 2006, pp. 58-60.

DOI: https://doi.org/10.15366/bp2019.22.011

Bajo Palabra. II Época. No 22. Pgs: 221-238 